OPEN ACCESS

Edited by:

Pedro Antonio Valdes-Sosa, University of Electronic Science and Technology of China, China

Reviewed by:

Bruce Graham,

University of Stirling, United Kingdom

Eduardo Martínez-Montes,

Cuban Neuroscience Center, Cuba

*Correspondence:

Anup Tuladhar

anup.tuladhar@ucalgary.ca

Received: 27 July 2021 Accepted: 21 October 2021 Published: 19 November 2021

Citation:

Tuladhar A, Moore JA, Ismail Z and Forkert ND (2021) Modeling Neurodegeneration in silico With

Deep Learning.

Front. Neuroinform. 15:748370. doi: 10.3389/fninf.2021.748370

\section{Modeling Neurodegeneration in silico With Deep Learning}

\author{
Anup Tuladhar ${ }^{1,2 *}$, Jasmine A. Moore ${ }^{1,2,3}$, Zahinoor Ismail ${ }^{2,4,5,6,7}$ and Nils D. Forkert ${ }^{1,2,4,8}$ \\ ${ }^{1}$ Department of Radiology, University of Calgary, Calgary, AB, Canada, ${ }^{2}$ Hotchkiss Brain Institute, University of Calgary, \\ Calgary, AB, Canada, ${ }^{3}$ Biomedical Engineering Program, University of Calgary, Calgary, AB, Canada, ${ }^{4}$ Department \\ of Clinical Neurosciences, University of Calgary, Calgary, AB, Canada, ${ }^{5}$ Department of Community Health Sciences, \\ University of Calgary, Calgary, AB, Canada, ${ }^{6}$ Department of Psychiatry, University of Calgary, Calgary, AB, Canada, ${ }^{7} \mathrm{O}^{\prime} \mathrm{Brien}$ \\ Institute for Public Health, University of Calgary, Calgary, AB, Canada, ${ }^{8}$ Alberta Children's Hospital Research Institute, \\ University of Calgary, Calgary, $A B$, Canada
}

Deep neural networks, inspired by information processing in the brain, can achieve human-like performance for various tasks. However, research efforts to use these networks as models of the brain have primarily focused on modeling healthy brain function so far. In this work, we propose a paradigm for modeling neural diseases in silico with deep learning and demonstrate its use in modeling posterior cortical atrophy (PCA), an atypical form of Alzheimer's disease affecting the visual cortex. We simulated PCA in deep convolutional neural networks (DCNNs) trained for visual object recognition by randomly injuring connections between artificial neurons. Results showed that injured networks progressively lost their object recognition capability. Simulated PCA impacted learned representations hierarchically, as networks lost object-level representations before category-level representations. Incorporating this paradigm in computational neuroscience will be essential for developing in silico models of the brain and neurological diseases. The paradigm can be expanded to incorporate elements of neural plasticity and to other cognitive domains such as motor control, auditory cognition, language processing, and decision making.

\footnotetext{
Keywords: deep neural networks (DNN), posterior cortical atrophy (PCA), neurodegenerative diseases, Alzheimer's disease, in silico simulation, visual object recognition, visual object agnosia, cognitive computational neuroscience
}

\section{INTRODUCTION}

By mimicking information processing and cognition in the human and primate brain (Kriegeskorte, 2015; Yamins and DiCarlo, 2016; Richards et al., 2019), deep neural networks have been shown to be capable of outperforming conventional machine learning methods for various classification and regression tasks, such as visual object recognition (LeCun et al., 2015; Vercio et al., 2020). Cognitive neuroscience continues to influence and advance the development of more biologically informed deep neural networks to further improve classification performance and achieve more human-like results (Kriegeskorte and Douglas, 2018). However, the potential for using deep neural networks as computational models of the human brain to advance our understanding of neurological diseases, such as Alzheimer's disease, has been largely neglected (Khatami et al., 2020). 
In this work, we propose a paradigm using deep neural networks as in silico models of neurodegeneration (Figure 1A). Within this context, in silico refers to employing computer models to improve our understanding of normal and pathological processes in the living organism. To date, in the context of using deep neural networks as models of normal cognitive function, this has been mostly studied using deep convolutional neural networks (DCNNs) with a focus on visual cognition (Cadieu et al., 2014; Khaligh-Razavi and Kriegeskorte, 2014; Yamins et al., 2014; Hong et al., 2016; Kheradpisheh et al., 2016; Horikawa and Kamitani, 2017; Wen et al., 2018; Grossman et al., 2019; Horikawa et al., 2019). The connectivity and hierarchical organization of DCNNs are inspired by the mammalian visual cortex, which make them promising biomimetic models for this purpose (Kriegeskorte, 2015; Yamins and DiCarlo, 2016; Peters and Kriegeskorte, 2021). DCNNs are state-of-the-art models for the prediction of neural responses of the ventral visual stream, particularly in higher order regions such as visual area V4 and inferior temporal cortex, during visual object recognition (Cadieu et al., 2014; Yamins et al., 2014). Additionally, it has been shown that the internal representation of objects (Cadieu et al., 2014; Khaligh-Razavi and Kriegeskorte, 2014) and faces (Grossman et al., 2019) in DCNNs are similar to the internal representations in humans. Consequently, current efforts aim to quantify the similarity of different DCNN architectures to the human brain through integrative benchmarking, such as the Brain-Score (Schrimpf et al., 2020).

Prior work in modeling neurological diseases have primarily explored computational models of cellular processes focusing on pathological changes such as excitotoxicity or abnormal bioenergetics (Le Masson et al., 2014; Muddapu et al., 2019), or connectome models based on structural or functional connectivity in the brain (Hof et al., 1997; Raj et al., 2012; Zhou et al., 2012; Ortiz et al., 2015; Peraza-Goicolea et al., 2020; Vanasse et al., 2021). Early work investigating structural or functional connectomes primarily focused on modeling specific aspects of disease progression, such as diffusive spread of misfolded tau and beta amyloids (Raj et al., 2012) or changes in network connectivity contributing to disease vulnerability or diagnosis (Zhou et al., 2012; Ortiz et al., 2015). More recent work has begun using the connectome to simulate disease states in silico, for example by modifying connection weights in a simulated functional connectome to predict changes in functional activation and connectivity in the brain (Peraza-Goicolea et al., 2020). A meta-analysis of the relationship between structural pathology and behavioral pathology supported the notion that network degeneration is a contributing factor to disease pathology (Vanasse et al., 2021). However, none of the aforementioned approaches used models that can perform tasks at near-human levels (LeCun et al., 2015; Vercio et al., 2020). Doing so would enable the simulation of changes in model behavior with disease progression in silico.

Deep neural network-based approaches have largely focused on data-driven analysis (Khatami et al., 2020), such as disease diagnosis or progression prediction (Lee et al., 2019; MartinezMurcia et al., 2019). One notable exception is the work by Lusch et al. (2018) who used a DCNN to model traumatic brain injury (TBI) as focal axonal swellings that cause spike train blockage. In these analyses, TBI was simulated by ablating DCNN weights. The work by Lusch et al. (2018) focused primarily on abruptly injuring the DCNN, with a very limited investigation of cumulative network injury, and did not quantify changes in artificial neuron activation patterns or representations. Additionally, the similarity of the DCNNs used in the study to biological neural networks, and thus their validity as brain-like models of visual cognition, were not investigated (Schrimpf et al., 2020).

In this study, we used DCNNs trained for visual object recognition to model posterior cortical atrophy (PCA), an atypical form of Alzheimer's marked by lesions in the visual cortex and attendant visual cognition deficits such as visual agnosia (Tang-Wai et al., 2004; Crutch et al., 2012; Migliaccio et al., 2016; Silva et al., 2017). Specifically, we trained the VGG-19 DCNN (Simonyan and Zisserman, 2014) to perform visual object recognition on the CIFAR-100 dataset (Krizhevsky, 2009). VGG-19 has one of the highest similarities to biological neural networks amongst feed-forward DCNNs as measured by the Brain-Score (Schrimpf et al., 2020). Trained networks underwent neurodegeneration, where connections between artificial neurons, mimicking biological synapses, were progressively lesioned. At each stage of simulated PCA we evaluated the respective changes in the injured network's object classification performance and activation patterns of its neurons.

\section{MATERIALS AND METHODS}

\section{Dataset and Model Training}

We trained the VGG-19 DCNN (Simonyan and Zisserman, 2014) to perform visual object recognition on the CIFAR-100 dataset (Krizhevsky, 2009). CIFAR-100 is a publicly available natural images dataset which contains 50,000 training and 10,000 testing images $(32 \times 32$ pixels $)$ of objects from 100 classes (e.g., roses, butterflies, and bicycles) organized into 20 categorical superclasses (e.g., flowers, insects, and vehicles).

The VGG-19 DCNN has 16 convolutional layers followed by two fully-connected dense layers with 1,000 neurons each (Figure 1B). The network's output layer has 100 neurons and classifies the input image into one of the 100 classes using the softmax function. The convolutional layers were initialized with weights pretrained on ImageNet (Deng et al., 2009) in order to make use of the convolutional kernel features learned on this much larger database. Input images were upscaled $4 \times$ to $128 \times 128$ pixels and the fully-connected dense and output layers were randomly initialized.

Dropout regularization (30\%) was used after each fullyconnected dense layer ("Dense 1" and "Dense 2," Figure 1B). This regularization technique helps reduce network overfitting to the training data and improve generalization performance of the uninjured baseline networks (Srivastava et al., 2014). Briefly, in each training round, $30 \%$ of the neurons and associated weights in the fully-connected dense layers were temporarily removed during training. The neurons and associated weights are restored after the training round and in the final, fully-trained, baseline uninjured model. 

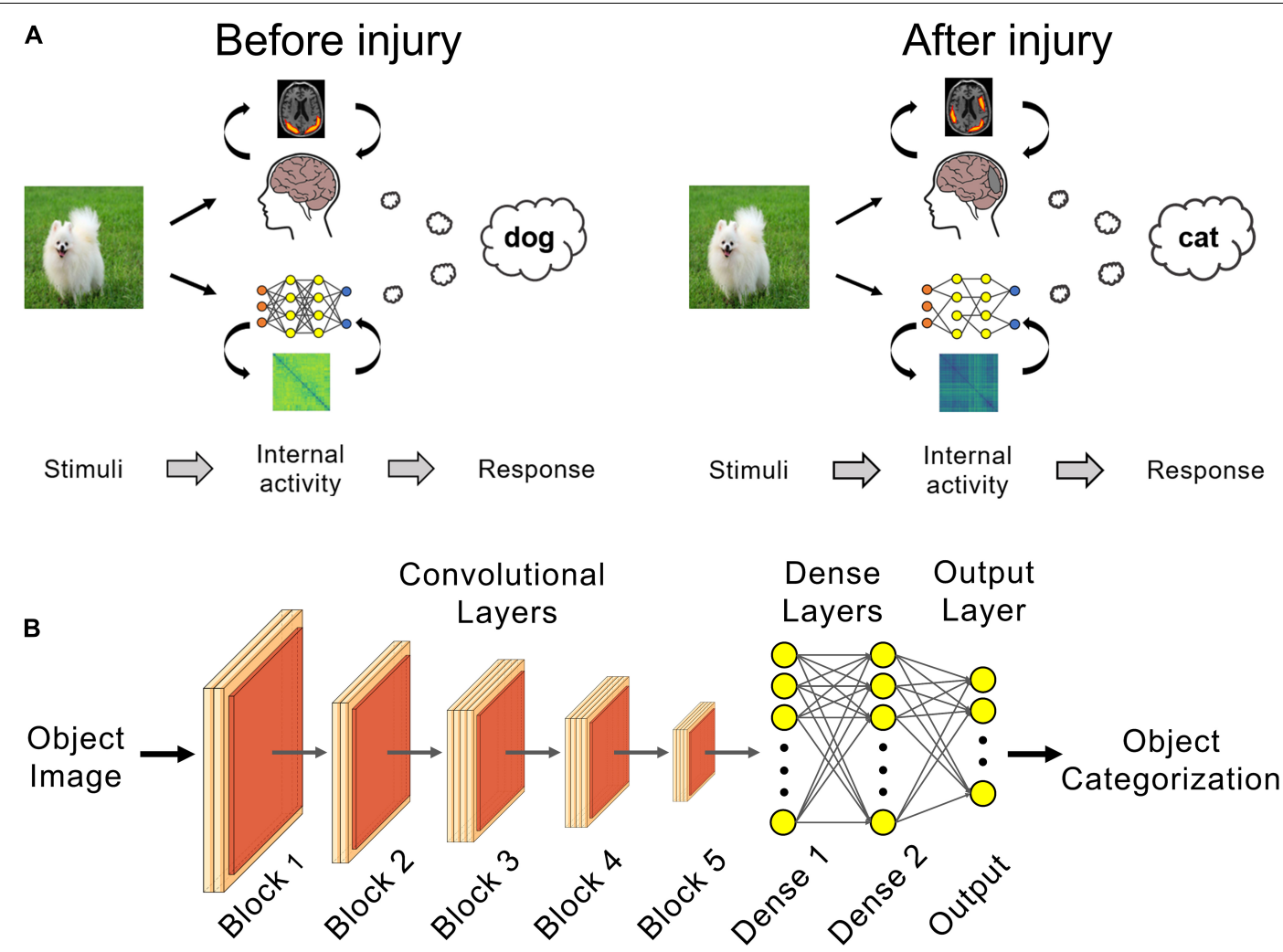

FIGURE 1 | Deep learning can be used as in silico models of brain injury. (A) Changes in internal patterns of activity between neurons and in external behavior can be studied in trained networks before and after a simulated neural injury, such as for posterior cortical atrophy (PCA) as shown here. (B) VGG 19 networks trained for visual object recognition on the CIFAR-100 dataset were used for PCA simulation experiments.

Training parameters resulting in the best network performance were optimized for the final model in preliminary investigations. More precisely, a fixed learning rate of $3 \times 10^{-5}$ was used with the RMSprop optimizer for 40 training epochs. The network was trained end-to-end, such that both the pretrained convolutional layers and the randomly initialized fully-connected dense and output layers were updated during training. This training was repeated with different random initializations of the fully-connected dense and output classification layers to produce 25 unique uninjured networks for use in subsequent experiments on simulating PCA injury. The uninjured networks produced at the end of training were used in these experiments regardless of training performances. Though no exclusion criterion was applied to uninjured networks after training, the use of 25 unique networks reduces potential bias from single outliers. The uninjured networks achieved an average test set performance of $76.48 \%$ (standard deviation: $0.53 \%$ ). Importantly, the networks were only given the class labels during training and no information about an object's superclass.

\section{Simulating Posterior Cortical Atrophy}

The trained networks underwent simulated PCA injury by randomly setting $\mathrm{x} \%$ of the network weights to zero, effectively severing the connections between artificial neurons. This was done for trained weights between all layers in a network, i.e., the weights between all the convolutional layers, dense layers and output layer (Figure 1B). We simulated the visual agnosia seen in PCA by repeatedly injuring each network at $0.1 \%$ increments such that the damage was cumulative. In other words, for each incremental injury, the previous injured connections remained at zero and an additional set of randomly selected connections are set to zero. The selection of weights to injure was random for each of the 25 trained networks, such that the course of degeneration was unique for each of the 25 networks.

\section{Quantifying Changes in Network Behavior}

After each injury, networks' accuracy in visual object recognition was measured on the set of test images. Random chance performance on visual object recognition was determined by calculating the probability of randomly selecting the correct class out of the 100 classes. As the number of test examples in each class is equal (100), the random chance performance is $1 \%$.

The network errors were further analyzed by evaluating the percentage of misclassified examples that were in the correct superclass, e.g., misidentifying a rose as another type of flower such as a tulip. This analysis was done on the test examples that the network made an incorrect prediction on. The incorrectly predicted class label was converted to the corresponding superclass label. This converted superclass label is an indirectly predicted superclass label, as the networks were never given information about the class-superclass structure of 
the data. The converted superclass label is compared to the true superclass label to determine if the network's incorrectly predicted class was within the correct superclass. This was calculated over all test set examples to obtain the percentage of errors that were within the correct superclass. The average level of errors within the correct superclass for the 25 baseline uninjured networks was $39.37 \%$ (standard deviation: $0.75 \%$ ). Random chance for network errors within the correct superclass was calculated based on the probability of selecting the correct superclass out of the 20 superclasses. As the number of test examples in each superclass is equal (500), the random chance performance is $5 \%$.

Representational similarity analysis (Kriegeskorte et al., 2008) was used to quantify changes in the learned internal representations of visual objects over the course of simulated PCA. Activations from neurons in a network's penultimate layer ("Dense 2," Figure 1B) were compared for pairs of test set visual stimuli using 1 - Pearson's correlation. This was done over all possible pairs of stimuli in the test set to create the representational dissimilarity matrices (RDMs), which provides an overview of a network's learned representation (Kriegeskorte et al., 2008; Khaligh-Razavi and Kriegeskorte, 2014; Yamins et al., 2014). The changes in a network's internal representation with injury was calculated by comparing the correlation of an injured network's RDM to the same model's uninjured RDM using Kendall's $\tau_{\mathrm{A}}$ correlation coefficient. Random noise levels for Kendall's $\tau_{\mathrm{A}}$ correlation coefficient were calculated as the Kendall's $\tau_{\mathrm{A}}$ of an uninjured network's RDM compared to a scrambled version of the same uninjured network's RDM. This was averaged across the uninjured RDMs for the 25 trained uninjured networks to obtain the random noise level of $0.005 \%$ for Kendall's $\tau_{\mathrm{A}}$.

\section{Statistical Analysis}

Object recognition performances, RDMs, and Kendall's $\tau_{\mathrm{A}}$ correlation coefficients were averaged across the 25 trained networks. Results were analyzed using PRISM (GraphPad, v9.0). Statistical significance was reported when $P<0.01$ and was determined using one-way repeated measures ANOVA or two-way ANOVA and Bonferroni's post hoc test for multiple comparisons, as appropriate.

\section{RESULTS}

Object recognition performance was already impaired by simulated PCA injury of just $0.2 \%$ of connections (Figure 2A). By $30 \%$ injury, the network performance was reduced to chance level (1\%). RDMs, used to analyze object representations within degenerating networks, showed that representations became more homogenous with injury (Figure 2B). Though object-level representations deteriorated markedly with injury, the networks retained some structured internal representations (Figure 2C). Even when networks lost all object recognition capability after $30 \%$ injury, the deteriorated internal representations retained correlation with the representations of uninjured networks and remained above the level of random noise $(0.005 \%)$.
Next, we investigated whether simulated PCA impacted the networks' representational capacity at all levels of semantic hierarchy equally by analyzing superclass-level RDMs. The corresponding results showed that trained networks were generally able to infer the existence of the categorical superclasses in the CIFAR-100 dataset, despite never have been given this information explicitly (Figure 3A). The internal representations of categorical superclass and broader image categories, such as animate vs. inanimate or natural vs. artificial, were better retained with injury compared to object-level representations (Figures $\mathbf{3 A}, \mathbf{B}$ ). This increased stability of superclass representation was also reflected in the manner in which networks made mistakes at the initial stages of simulated PCA, as objects were misclassified for another object within the same superclass (e.g., a rose is misclassified as another type of flower) at the same rate as uninjured networks until 1.3\% injury; thereafter, the networks made more random errors (Figure 3C).

\section{DISCUSSION}

Deep learning is a powerful biomimetic paradigm for modeling cognitive processes and information processing in the brain (Richards et al., 2019). Efforts thus far on using deep learning to study the human brain have primarily focused on modeling normal function (Cadieu et al., 2014; Khaligh-Razavi and Kriegeskorte, 2014; Yamins et al., 2014; Hong et al., 2016; Kheradpisheh et al., 2016; Horikawa and Kamitani, 2017; Kell et al., 2018; Pandarinath et al., 2018; Wen et al., 2018; Grossman et al., 2019; Horikawa et al., 2019; Botvinick et al., 2020; Hashemzadeh et al., 2020; Caucheteux et al., 2021). In this paper, we present an important new direction, namely using deep learning for modeling brain dysfunction in silico. As deep neural networks incorporate neurally mechanistic features to increase their biological plausibility, it will be important to investigate how well they model both normal function and disease if they are to become truly brain-like (Kriegeskorte, 2015; Yamins and DiCarlo, 2016; Kriegeskorte and Douglas, 2018; Richards et al., 2019).

Based on the simulated PCA in a DCNN, it was shown that increasing functional deficits were associated with greater levels of injury in our artificial neural networks, parallel to increased visual agnosia with greater atrophy (Hof et al., 1997; Fox et al., 1999; Zarei et al., 2013; Silva et al., 2017). At the initial stages of simulated PCA, the injured networks tended to misclassify objects as another similar item, akin to misidentifying a fork as a knife by patients with visual agnosia (Milner et al., 1991). Simulated neurodegeneration affected objectlevel representations more severely than broader categoricalrepresentations, similar to the loss of object-specific knowledge occurring prior to object category knowledge loss in patients with semantic dementia (Hodges et al., 1995).

This study is a first step in investigating the use of deep neural networks as in silico models of neural injury. As such, limitations on the current work and avenues for future investigations must be highlighted. Notably, the in silico model was more sensitive to injury compared to the human brain, showing measurable impairments after just $0.2 \%$ injury. In contrast, cognitive impairments do not typically manifest until much greater 

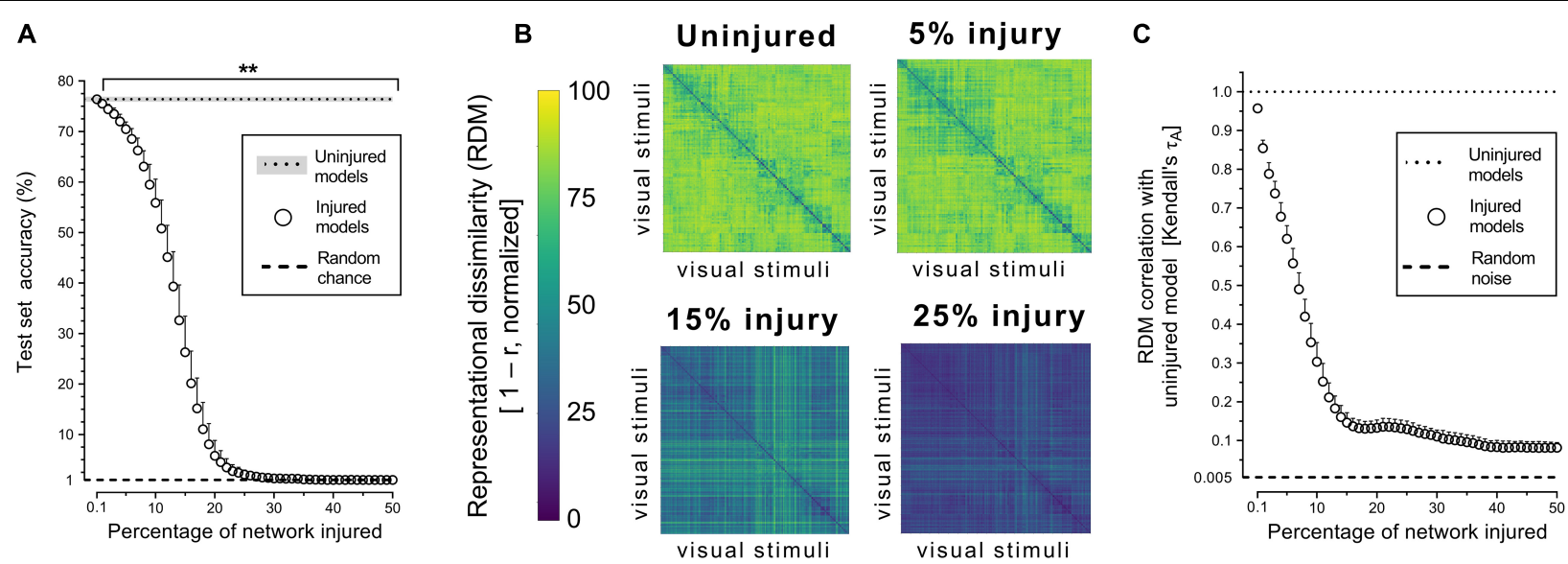

FIGURE 2 | Trained VGG-19 networks underwent progressive neurodegeneration. (A) Object recognition performance of injured VGG-19 models on the CIFAR-100 dataset with simulated PCA. (B) Internal activity of artificial neurons in the model's penultimate layer visualized with representational similarity analysis. The RDMs visualized are the average of the 25 trained networks. (C) Changes in internal activity patterns relative to uninjured control models measured using Kendall's $\tau_{A}$ correlation coefficient. Data are shown as mean $+\mathrm{SD}, n=25$ models, ${ }^{\star \star} P<0.01$ with one-way repeated measures ANOVA with Bonferroni's correction for multiple comparisons.

levels of cortical atrophy (Fox et al., 1999; Zarei et al., 2013). However, this must also take into consideration that clinical detection of PCA often occurs late in the disease course. PCA is easily misdiagnosed, with visual impairment interpreted as ophthalmologic or refractive problems, and cortical dysfunction considered only when cognitive impairment is more overt, resulting in accurate assessment and treatment (Crutch et al., 2012). Although a disease time course for PCA is not available, Lehmann et al. (2011) reported a 10-20\% decrease of cortical thickness in the occipitoparietal and occipitotemporal regions of patients diagnosed with PCA (Lehmann et al., 2011), with gray matter loss of approximately $2 \%$ of whole brain volume per year (Lehmann et al., 2012). PCA patients show a range of cognitive deficits, with one study reporting a $40-50 \%$ decrease in visual task performance compared to controls (McMonagle et al., 2006). This magnitude of deficit is comparable with the decrease in performance with $10-20 \%$ injury in our model, though more rigorous comparison between deficits in clinical and simulated PCA are needed.

Another limitation is that our simulation assumed a static system that does not change its connectivity over the course of PCA. In contrast, the brain is a dynamic system that undergoes synaptic plasticity and functional connectivity changes in response to the insult itself, as well as to rehabilitative and pharmacologic interventions (Voss et al., 2017). This plasticity could be added to our in silico model by retraining the damaged DCNNs in between inflicting injuries. While this is outside the scope of this preliminary study, research efforts on DCNN pruning may provide some insight on injured network retraining. Pruning is often undertaken to compress networks by selectively removing weights to reduce the size and computational demand of the networks, for example by removing low magnitude weights (Han et al., 2015). However, with sufficient pruning, the networks will eventually suffer large declines in performance. To mitigate this, the networks can be retrained, such as after pruning or over the course of progressive pruning (Mittal et al., 2019; Marcin and Maciej, 2020). It has been shown that this retraining allows the removal of a substantially larger number of connections while retaining comparable performance. Applying these retraining algorithms to the injured networks may provide a way to mimic neuroplasticity in silico, which will be essential to capturing the complexities of neural injury in more biologically plausible models.

Moreover, the current study used weight ablation to simulate progressive neurodegeneration in a DCNN. However, it remains to be seen if alternative injury types may be more appropriate for simulating PCA or other neurodegenerative diseases. The network weight ablation injury used here simulates synaptic injury, whereas cortical atrophy seen in PCA affects both neurons and synapses (Lehmann et al., 2011, 2012). Thus, alternative approaches to modeling could include network node ablation to simulate neuronal injury, or a combination of weight and network node ablation to simulate a combination of synaptic and neuronal injury. Furthermore, alternative network modifications, such as randomly rewiring network nodes or setting network weights to random values instead of zero (Gaier and $\mathrm{Ha}$, 2019; Xie et al., 2019), may also be studied to determine their appropriateness for modeling PCA or other types of dysfunction seen in neurodegenerative and neurological diseases.

Alternative courses of simulating the progressive injury seen in PCA and other neurodegenerative diseases may also be explored. For example, the current study used a sudden ablation injury of randomly selected weights across the entire network, with progressive injury simulated by increasing the number of ablated weights. An alternative approach could be to progressively decrease injured synapses to zero or add increasing noise to the weights. However, it remains to be seen whether this better recapitulates the slower manifestation of cognitive deficits seen clinically (Fox et al., 1999; Zarei et al., 2013). In contrast to random weight ablation, ablation 

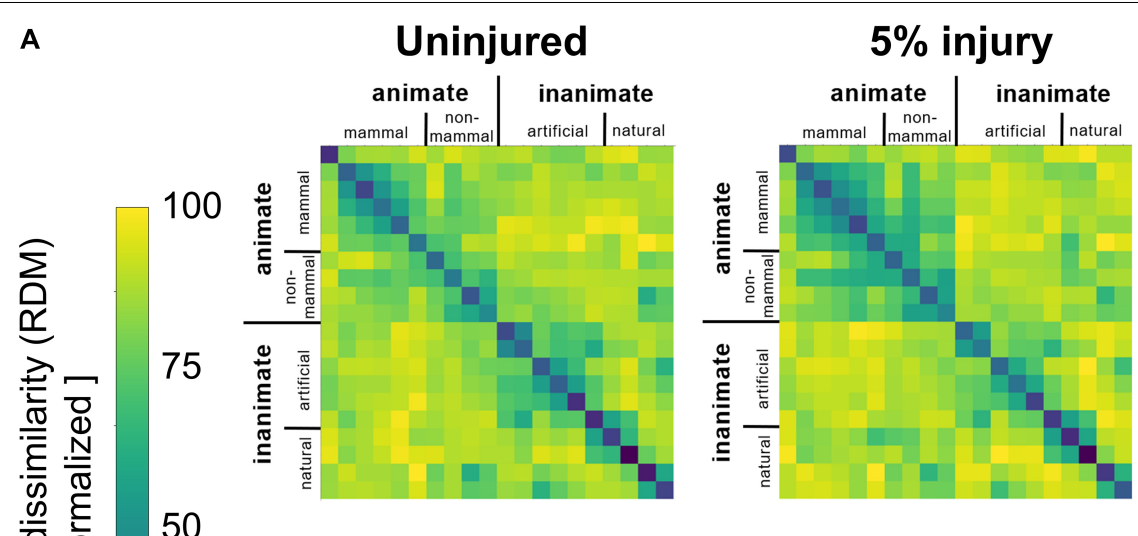

50
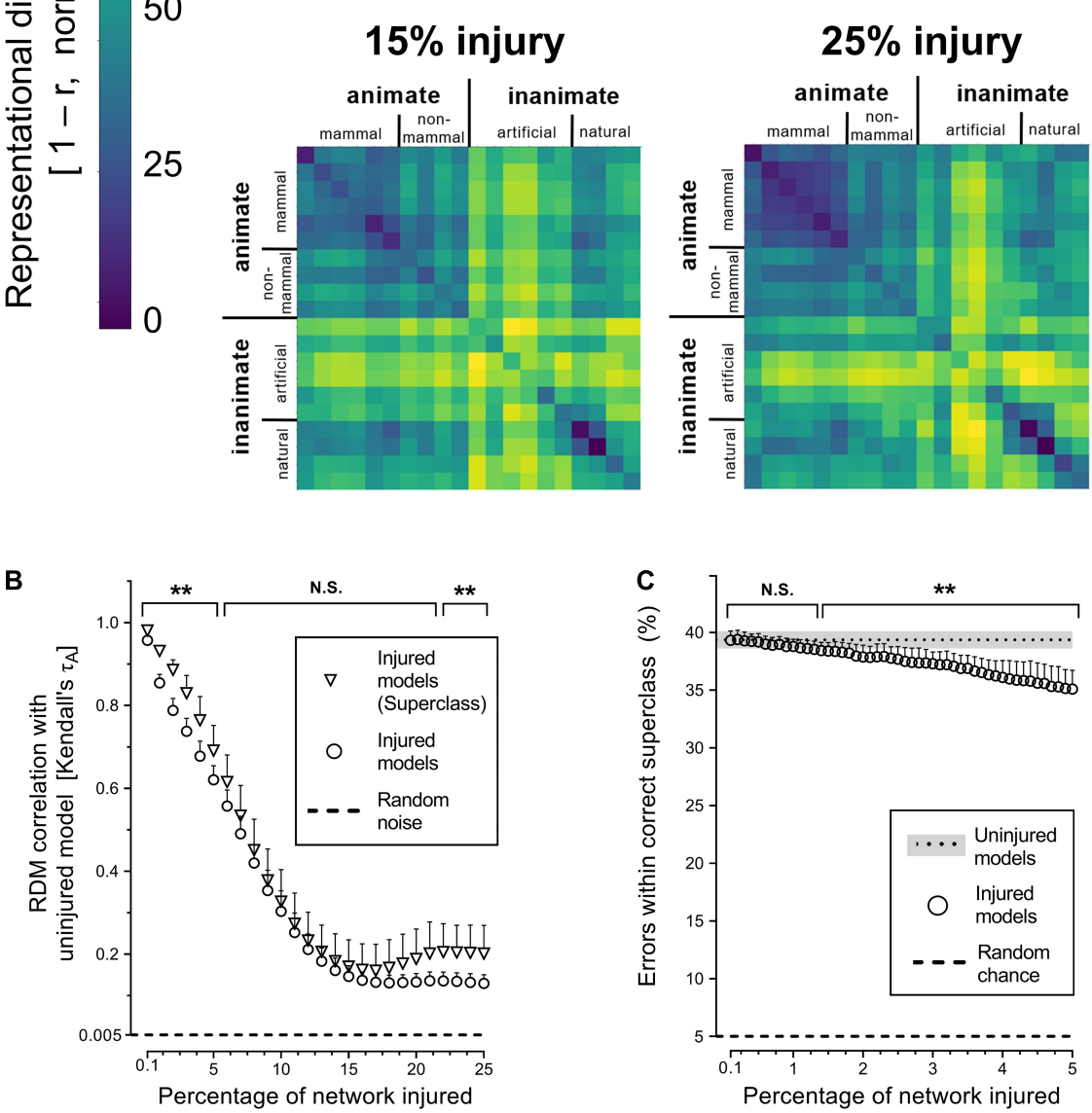

FIGURE 3 | Degenerating neural networks retained generalized information better than specific details. (A) Representational similarity of objects within a categorical superclass. The RDMs visualized are the average of the 25 trained networks. (B) Changes in internal activity patterns, relative to uninjured control models, at the object and superclass-level measured using Kendall's $\tau_{\mathrm{A}}$ correlation coefficient. (C) Percentage of misclassified objects that were within the correct categorical superclass. Data are shown as mean $+\mathrm{SD}, n=25$ models, ${ }^{\star \star} P<0.01$ using two-way ANOVA (B) or one-way repeated measures ANOVA (C) with Bonferroni's correction for multiple comparisons.

of connections based on their strengths such as in network pruning (Han et al., 2015; Mittal et al., 2019; Marcin and Maciej, 2020), or specifically targeting excitatory (positive) or inhibitory (negative) connections (Song et al., 2016; Mackwood et al., 2021) may be instructive.

Future work may also investigate more biologically informed methods for modeling disease. One possibility may be to model the diffusive spread of disease-causing agents such as misfolded tau and beta amyloids across artificial neurons and synapses in the deep neural network (Raj et al., 2012; Vogel et al., 2021). Another approach may be to injure individual DCNN layers based on their correspondence to regions of the ventral visual stream such as V4 and inferior temporal cortex (Cadieu et al., 2014; Yamins et al., 2014). This layer-wise injury could be informed by neuroimaging, such as patterns of atrophy in PCA (Whitwell et al., 2007; Lehmann et al., 2011, 2012). 
Finally, this paradigm of simulating neural injury can be extended to deep neural networks in other cognitive domains such as motor control (Pandarinath et al., 2018), auditory cognition (Kell et al., 2018), language processing (Hashemzadeh et al., 2020; Caucheteux et al., 2021), memory (Schapiro et al., 2017), and decision making (Botvinick et al., 2020). This may enable the development of in silico models of other neurological diseases, such as Parkinson's disease, and the study of their impact on multiple cognitive systems.

In conclusion, our results show that deep neural networks may serve as in silico models of neurodegeneration, as the injured network's behavior is similar to that seen clinically. Future work will need to study this correspondence with clinical data in more detail. Marrying in silico disease modeling with clinical data may enable the creation of patient-specific computational models, and may be integral to precision medicine for neurological diseases.

\section{DATA AVAILABILITY STATEMENT}

The CIFAR-100 dataset analyzed for this study is publicly available at https://www.cs.toronto.edu/ kriz/cifar.html. The

\section{REFERENCES}

Botvinick, M., Wang, J. X., Dabney, W., Miller, K. J., and Kurth-Nelson, Z. (2020). Deep reinforcement learning and its neuroscientific implications. Neuron 107, 603-616. doi: 10.1016/j.neuron.2020.06.014

Cadieu, C. F., Hong, H., Yamins, D. L. K., Pinto, N., Ardila, D., Solomon, E. A., et al. (2014). Deep neural networks rival the representation of primate IT cortex for core visual object recognition. PLoS Comput. Biol. 10:e1003963. doi: 10.1371/journal.pcbi.1003963

Caucheteux, C., Gramfort, A., and King, J.-R. (2021). Disentangling syntax and semantics in the brain with deep networks. Arxiv arxiv.org/pdf/2103.01620.pdf

Crutch, S. J., Lehmann, M., Schott, J. M., Rabinovici, G. D., Rossor, M. N., and Fox, N. C. (2012). Posterior cortical atrophy. Lancet Neurol. 11, 170-178. doi: 10.1016/s1474-4422(11)70289-7

Deng, J., Dong, W., Socher, R., Li, L.-J., Li, K., and Fei-Fei, L. (2009). "ImageNet: a large-scale hierarchical image database," in Proceedings of the 2009 IEEEE Conference on Computer Vision and Pattern Recognition, Miami, FL, 248-255.

Fox, N. C., Scahill, R. I., Crum, W. R., and Rossor, M. N. (1999). Correlation between rates of brain atrophy and cognitive decline in AD. Neurology 52, 1687-1689. doi: 10.1212/wnl.52.8.1687

Gaier, A., and Ha, D. (2019). Weight agnostic neural networks. Arxiv arxiv.org/abs/1906.04358

Grossman, S., Gaziv, G., Yeagle, E. M., Harel, M., Mégevand, P., Groppe, D. M., et al. (2019). Convergent evolution of face spaces across human face-selective neuronal groups and deep convolutional networks. Nat. Commun. 10:4934. doi: 10.1038/s41467-019-12623-6

Han, S., Pool, J., Tran, J., and Dally, W. J. (2015). Learning both weights and connections for efficient neural networks. Arxiv

Hashemzadeh, M., Kaufeld, G., White, M., Martin, A. E., and Fyshe, A. (2020). From language to language-ish: how brain-like is an LSTM's representation of nonsensical language stimuli? Arxiv

Hodges, J. R., Graham, N., and Patterson, K. (1995). Charting the progression in semantic dementia: implications for the organisation of semantic memory. Memory 3, 463-495. doi: 10.1080/09658219508253161

Hof, P. R., Vogt, B. A., Bouras, C., and Morrison, J. H. (1997). Atypical form of Alzheimer's disease with prominent posterior cortical atrophy: a review of lesion distribution and circuit disconnection in cortical visual pathways. Vis. Res. 37, 3609-3625. doi: 10.1016/s0042-6989(96)00240-4

Hong, H., Yamins, D. L. K., Majaj, N. J., and DiCarlo, J. J. (2016). Explicit information for category-orthogonal object properties increases along the ventral stream. Nat. Neurosci. 19, 613-622. doi: 10.1038/nn.4247 pretrained VGG-19 used for this study is available from Tensorflow 2.0. The source code for fine-tuning pretrained VGG-19 models on CIFAR-100, for injuring models, and for evaluating their performance and RDMs are available from the corresponding author upon request.

\section{AUTHOR CONTRIBUTIONS}

AT, JM, and NF designed the study. AT and JM developed the computer code. AT performed the research, data analysis and drafted the manuscript. All authors revised the manuscript and approved the submitted version.

\section{FUNDING}

This work was supported by the Natural Sciences and Engineering Research Council of Canada (AT, JM, and NF), Brain Create (JM), Alberta Innovates and Alberta Advanced Education (JM), Canada Research Chairs Program (NF), and River Fund at Calgary Foundation (NF).

Horikawa, T., Aoki, S. C., Tsukamoto, M., and Kamitani, Y. (2019). Characterization of deep neural network features by decodability from human brain activity. Sci. Data 6:190012. doi: 10.1038/sdata.2019.12

Horikawa, T., and Kamitani, Y. (2017). Generic decoding of seen and imagined objects using hierarchical visual features. Nat. Commun. 8:15037. doi: 10.1038/ ncomms 15037

Kell, A. J. E., Yamins, D. L. K., Shook, E. N., Norman-Haignere, S. V., and McDermott, J. H. (2018). A task-optimized neural network replicates human auditory behavior, predicts brain responses, and reveals a cortical processing hierarchy. Neuron 98, 630.e16-644.e16. doi: 10.1016/j.neuron.2018.03.044

Khaligh-Razavi, S.-M., and Kriegeskorte, N. (2014). Deep supervised, but not unsupervised, models may explain IT cortical representation. PLoS Comput. Biol. 10:e1003915. doi: 10.1371/journal.pcbi.1003915

Khatami, S. G., Mubeen, S., and Hofmann-Apitius, M. (2020). Data science in neurodegenerative disease: its capabilities, limitations, and perspectives. Curr. Opin. Neurol. 33, 249-254. doi: 10.1097/wco.0000000000000795

Kheradpisheh, S. R., Ghodrati, M., Ganjtabesh, M., and Masquelier, T. (2016). Deep networks can resemble human feed-forward vision in invariant object recognition. Sci. Rep. 6:32672. doi: 10.1038/srep32672

Kriegeskorte, N. (2015). Deep neural networks: a new framework for modeling biological vision and brain information processing. Annu. Rev. Vis. Sci. 1, 417-446. doi: 10.1146/annurev-vision-082114-035447

Kriegeskorte, N., and Douglas, P. K. (2018). Cognitive computational neuroscience. Nat. Neurosci. 21, 1148-1160. doi: 10.1038/s41593-018-0210-5

Kriegeskorte, N., Mur, M., and Bandettini, P. A. (2008). Representational similarity analysis - connecting the branches of systems neuroscience. Front. Syst. Neurosci. 2:4. doi: 10.3389/neuro.06.004.2008

Krizhevsky, A. (2009). Learning Multiple Layers of Features From Tiny Images: Technical Report TR-2009. Toronto, ON: University of Toronto.

Le Masson, G., Przedborski, S., and Abbott, L. F. (2014). A computational model of motor neuron degeneration. Neuron 83, 975-988. doi: 10.1016/j.neuron.2014. 07.001

LeCun, Y., Bengio, Y., and Hinton, G. (2015). Deep learning. Nature 521, 436-444. doi: 10.1038/nature14539

Lee, G., Nho, K., Kang, B., Sohn, K.-A., Kim, D., Weiner, M. W., et al. (2019). Predicting Alzheimer's disease progression using multi-modal deep learning approach. Sci. Rep. 9:1952. doi: 10.1038/s41598-018-37769-z

Lehmann, M., Barnes, J., Ridgway, G. R., Ryan, N. S., Warrington, E. K., Crutch, S. J., et al. (2012). Global gray matter changes in posterior cortical atrophy: a serial imaging study. Alzheimer's Dement. 8, 502-512. doi: 10.1016/j.jalz.2011. 09.225 
Lehmann, M., Crutch, S. J., Ridgway, G. R., Ridha, B. H., Barnes, J., Warrington, E. K., et al. (2011). Cortical thickness and voxel-based morphometry in posterior cortical atrophy and typical Alzheimer's disease. Neurobiol. Aging 32, 1466-1476. doi: 10.1016/j.neurobiolaging.2009.08.017

Lusch, B., Weholt, J., Maia, P. D., and Kutz, J. N. (2018). Modeling cognitive deficits following neurodegenerative diseases and traumatic brain injuries with deep convolutional neural networks. Brain Cogn. 123, 154-164. doi: 10.1016/j.bandc. 2018.02.012

Mackwood, O., Naumann, L. B., and Sprekeler, H. (2021). Learning excitatoryinhibitory neuronal assemblies in recurrent networks. eLife 10:e59715. doi: 10.7554/elife.59715

Marcin, P., and Maciej, W. (2020). "Retrain or not retrain? - Efficient pruning methods of deep CNN networks," in Lecture Notes in Computer Science eds V. V. Krzhizhanovskaya, G. Závodszky, M. H. Lees, J. J. Dongarra, P. M. A. Sloot, S. Brissos, et al. (Berlin: Springer International Publishing), 452-463. doi: 10.1007/978-3-030-50420-5_34

Martinez-Murcia, F. J., Ortiz, A., Gorriz, J.-M., Ramirez, J., and Castillo-Barnes, D. (2019). Studying the manifold structure of Alzheimer's disease: a deep learning approach using convolutional autoencoders. IEEE J. Biomed. Health 24, 17-26. doi: 10.1109/jbhi.2019.2914970

McMonagle, P., Deering, F., Berliner, Y., and Kertesz, A. (2006). The cognitive profile of posterior cortical atrophy. Neurology 66, 331-338. doi: 10.1212/01. wnl.0000196477.78548.db

Migliaccio, R., Gallea, C., Kas, A., Perlbarg, V., Samri, D., Trotta, L., et al. (2016). Functional connectivity of ventral and dorsal visual streams in posterior cortical atrophy. J. Alzheimer's Dis. 51, 1119-1130. doi: 10.3233/jad-150934

Milner, A. D., Perrett, D. I., Johnston, R. S., Benson, P. J., Jordan, T. R., Heeley, D. W., et al. (1991). Perception and action in "visual form agnosia.". Brain 114, 405-428. doi: 10.1093/brain/114.1.405

Mittal, D., Bhardwaj, S., Khapra, M. M., and Ravindran, B. (2019). Studying the plasticity in deep convolutional neural networks using random pruning. Mach. Vis. Appl. 30, 203-216. doi: 10.1007/s00138-018-01001-9

Muddapu, V. R., Mandali, A., Chakravarthy, V. S., and Ramaswamy, S. (2019). A computational model of loss of dopaminergic cells in parkinson's disease due to glutamate-induced excitotoxicity. Front. Neural Circ. 13:11. doi: 10.3389/fncir. 2019.00011

Ortiz, A., Munilla, J., Álvarez-Illán, I., Górriz, J. M., Ramírez, J., and Initiative, A. D. N. (2015). Exploratory graphical models of functional and structural connectivity patterns for Alzheimer's disease diagnosis. Front. Comput. Neurosci. 9:132. doi: 10.3389/fncom.2015.00132

Pandarinath, C., O'Shea, D. J., Collins, J., Jozefowicz, R., Stavisky, S. D., Kao, J. C., et al. (2018). Inferring single-trial neural population dynamics using sequential auto-encoders. Nat. Methods 15, 805-815. doi: 10.1038/s41592-018-0109-9

Peraza-Goicolea, J. A., Martínez-Montes, E., Aubert, E., Valdés-Hernández, P. A., and Mulet, R. (2020). Modeling functional resting-state brain networks through neural message passing on the human connectome. Neural Netw. 123, 52-69. doi: 10.1016/j.neunet.2019.11.014

Peters, B., and Kriegeskorte, N. (2021). Capturing the objects of vision with neural networks. Nat. Hum. Behav. 5, 1127-1144. doi: 10.1038/s41562-021-01194-6

Raj, A., Kuceyeski, A., and Weiner, M. (2012). A network diffusion model of disease progression in dementia. Neuron 73, 1204-1215. doi: 10.1016/j.neuron.2011.12.040

Richards, B. A., Lillicrap, T. P., Beaudoin, P., Bengio, Y., Bogacz, R., Christensen, A., et al. (2019). A deep learning framework for neuroscience. Nat. Neurosci. 22, 1761-1770. doi: 10.1038/s41593-019-0520-2

Schapiro, A. C., Turk-Browne, N. B., Botvinick, M. M., and Norman, K. A. (2017). Complementary learning systems within the hippocampus: a neural network modelling approach to reconciling episodic memory with statistical learning. Philos. Trans. R. Soc. B Biol. Sci. 372:20160049. doi: 10.1098/rstb.2016.0049

Schrimpf, M., Kubilius, J., Lee, M. J., Murty, N. A. R., Ajemian, R., and DiCarlo, J. J. (2020). Integrative benchmarking to advance neurally mechanistic models of human intelligence. Neuron 108, 413-423. doi: 10.1016/j.neuron.2020.07.040

Silva, M. N. M., da, Millington, R. S., Bridge, H., James-Galton, M., and Plant, G. T. (2017). Visual dysfunction in posterior cortical atrophy. Front. Neurol. 8:389. doi: 10.3389/fneur.2017.00389
Simonyan, K., and Zisserman, A. (2014). Very deep convolutional networks for large-scale image recognition. Arxiv Available online at: arxiv.org/abs/1409.1556

Song, H. F., Yang, G. R., and Wang, X.-J. (2016). Training excitatory-inhibitory recurrent neural networks for cognitive tasks: a simple and flexible framework. PLoS Comput. Biol. 12:e1004792. doi: 10.1371/journal.pcbi.1004792

Srivastava, N., Hinton, G., Krizhevsky, A., Sutskever, I., and Salakhutdinov, R. (2014). Dropout: a simple way to prevent neural networks from overfitting. J. Mach. Learn. Res. 15, 1929-1958.

Tang-Wai, D. F., Graff-Radford, N. R., Boeve, B. F., Dickson, D. W., Parisi, J. E., Crook, R., et al. (2004). Clinical, genetic, and neuropathologic characteristics of posterior cortical atrophy. Neurology 63, 1168-1174. doi: 10.1212/01.wnl. 0000140289.18472 .15

Vanasse, T. J., Fox, P. T., Fox, P. M., Cauda, F., Costa, T., Smith, S. M., et al. (2021). Brain pathology recapitulates physiology: a network meta-analysis. Commun. Biol. 4:301. doi: 10.1038/s42003-021-01832-9

Vercio, L. L., Amador, K., Bannister, J. J., Crites, S., Gutierrez, A., MacDonald, M. E., et al. (2020). Supervised machine learning tools: a tutorial for clinicians. J. Neural Eng. 17:062001. doi: 10.1088/1741-2552/abbff2

Vogel, J. W., Young, A. L., Oxtoby, N. P., Smith, R., Ossenkoppele, R., Strandberg, O. T., et al. (2021). Four distinct trajectories of tau deposition identified in Alzheimer's disease. Nat. Med. 27, 871-881. doi: 10.1038/s41591-021-01309-6

Voss, P., Thomas, M. E., Cisneros-Franco, J. M., and de Villers-Sidani, É (2017). Dynamic brains and the changing rules of neuroplasticity: implications for learning and recovery. Front. Psychol. 08:1657. doi: 10.3389/fpsyg.2017.01657

Wen, H., Shi, J., Chen, W., and Liu, Z. (2018). Deep residual network predicts cortical representation and organization of visual features for rapid categorization. Sci. Rep. 8:3752. doi: 10.1038/s41598-018-22160-9

Whitwell, J. L., Jack, C. R., Kantarci, K., Weigand, S. D., Boeve, B. F., Knopman, D. S., et al. (2007). Imaging correlates of posterior cortical atrophy. Neurobiol. Aging 28, 1051-1061. doi: 10.1016/j.neurobiolaging.2006.05.026

Xie, S., Kirillov, A., Girshick, R., and He, K. (2019). Exploring randomly wired neural networks for image recognition. Arxiv Available online at: arxiv.org/abs/1904.01569

Yamins, D. L. K., and DiCarlo, J. J. (2016). Using goal-driven deep learning models to understand sensory cortex. Nat. Neurosci. 19, 356-365. doi: 10.1038/nn.4244

Yamins, D. L. K., Hong, H., Cadieu, C. F., Solomon, E. A., Seibert, D., and DiCarlo, J. J. (2014). Performance-optimized hierarchical models predict neural responses in higher visual cortex. Proc. Natl. Acad. Sci. U.S.A. 111, 8619-8624. doi: 10.1073/pnas.1403112111

Zarei, M., Ibarretxe-Bilbao, N., Compta, Y., Hough, M., Junque, C., Bargallo, N., et al. (2013). Cortical thinning is associated with disease stages and dementia in Parkinson's disease. J. Neurol. Neurosurg. Psychiatry 84, 875. doi: 10.1136/jnnp2012-304126

Zhou, J., Gennatas, E. D., Kramer, J. H., Miller, B. L., and Seeley, W. W. (2012). Predicting regional neurodegeneration from the healthy brain functional connectome. Neuron 73, 1216-1227. doi: 10.1016/j.neuron.2012.03.004

Conflict of Interest: The authors declare that the research was conducted in the absence of any commercial or financial relationships that could be construed as a potential conflict of interest.

Publisher's Note: All claims expressed in this article are solely those of the authors and do not necessarily represent those of their affiliated organizations, or those of the publisher, the editors and the reviewers. Any product that may be evaluated in this article, or claim that may be made by its manufacturer, is not guaranteed or endorsed by the publisher.

Copyright $\odot 2021$ Tuladhar, Moore, Ismail and Forkert. This is an open-access article distributed under the terms of the Creative Commons Attribution License (CC BY). The use, distribution or reproduction in other forums is permitted, provided the original author(s) and the copyright owner(s) are credited and that the original publication in this journal is cited, in accordance with accepted academic practice. No use, distribution or reproduction is permitted which does not comply with these terms. 